\title{
Cosmic Antiproton Constraints on Effective Interactions of the Dark Matter
}

\author{
Kingman Cheung ${ }^{1,2,3}$, Po-Yan Tseng ${ }^{2}$, and Tzu-Chiang Yuan ${ }^{4}$ \\ ${ }^{1}$ Division of Quantum Phases $\&$ Devices, \\ School of Physics, Konkuk university, Seoul 143-701, Korea \\ ${ }^{2}$ Department of Physics, National Tsing Hua University, Hsinchu 300, Taiwan \\ ${ }^{3}$ Physics Division, National Center for Theoretical Sciences, Hsinchu 300, Taiwan \\ ${ }^{4}$ Institute of Physics, Academia Sinica, Nankang, Taipei 11529, Taiwan
}

(Dated: June 30, 2018)

\begin{abstract}
Using an effective interaction approach to describe the interactions between the dark matter particle and the light degrees of freedom of the standard model, we calculate the antiproton flux due to the annihilation of the dark matter in the Galactic Halo and compare to the most recent antiproton spectrum of the PAMELA experiment. We obtain useful constraints on the size of the effective interactions that are comparable to those deduced from collider and gamma-ray experiments.
\end{abstract}




\section{INTRODUCTION}

The presence of cold dark matter (CDM) in our Universe is now well established by a number of observational experiments, especially the very precise measurement of the cosmic microwave background radiation in the Wilkinson Microwave Anisotropy Probe (WMAP) experiment [1]. The measured value of the CDM relic density is

$$
\Omega_{\mathrm{CDM}} h^{2}=0.1099 \pm 0.0062
$$

where $h$ is the Hubble constant in units of $100 \mathrm{~km} / \mathrm{Mpc} / \mathrm{s}$. Though the gravitation nature of the dark matter is established, we know almost nothing about its particle nature, except that it is nonbaryonic and to a high extent electrically neutral.

One of the most appealing and natural CDM particle candidates is weakly-interacting massive particle (WIMP). It is a coincidence that if the dark matter (DM) is thermally produced in the early Universe, the required annihilation cross section is right at the order of weak interaction. The relation between the relic density and the thermal annihilation cross section can be given by the following simple formula [2]

$$
\Omega_{\chi} h^{2} \simeq \frac{0.1 \mathrm{pb}}{\langle\sigma v\rangle}
$$

where $\langle\sigma v\rangle$ is the annihilation rate of the dark matter around the time of freeze-out. Given the measured $\Omega_{\mathrm{CDM}} h^{2}$ the annihilation rate is about $1 \mathrm{pb}$ or $10^{-26} \mathrm{~cm}^{3} \mathrm{~s}^{-1}$. This is exactly the size of the cross section that one expects from a weak interaction process and that would also give a large to moderate production rate of the WIMP at the Large Hadron Collider (LHC). In general, production of dark matter at the LHC would give rise to a large missing energy. Thus, the anticipated signature in the final state is high- $p_{T}$ jets or leptons plus a large missing energy. Note that there could be nonthermal sources for the dark matter, such as decay from exotic relics like moduli fields, cosmic strings, etc. In such cases, the annihilation rate in Eq. (11) can be larger than the value quoted above.

There have been many proposed candidates for the dark matter. Instead of specifying a particular model we adopt an effective interaction approach to describe the interactions of the dark matter particle with the standard model (SM) particles [3-8]. One simple realization is that the dark matter particle exists in a hidden sector, which communicates to the SM sector via a heavy degree of freedom in the connector sector. At energy scale well below this heavy 
mediator the interactions can be conveniently described by a set of effective interactions. The strength of each interaction depends on the nature of the dark matter particle and the mediator. An interesting set of interactions between the fermionic dark matter $\chi$ and the light quarks $q$ can be described by $(\bar{q} \Gamma q)\left(\bar{\chi} \Gamma^{\prime} \chi\right)$, where $\Gamma, \Gamma^{\prime}=\sigma^{\mu \nu}, \sigma^{\mu \nu} \gamma^{5}, \gamma^{\mu}, \gamma^{\mu} \gamma^{5}, \gamma^{5}$ and 1. Note that due to the following identity

$$
\sigma^{\mu \nu} \gamma^{5}=\frac{i}{2} \epsilon^{\mu \nu \alpha \beta} \sigma_{\alpha \beta}
$$

the axial tensor $\sigma^{\mu \nu} \gamma^{5}$ is related to the tensor $\sigma^{\alpha \beta}$ and thus should not be regarded as an independent set. A more complete set of interactions involving fermionic and scalar dark matter candidates that we will study in this work are listed in Table I. Without a particular model in mind we will treat each interaction independently in our analysis.

There have been some recent works on constraining the interactions at present and future collider experiments [3 -5] and using gamma-ray experiments [6]. Fan et al. [7] also wrote down the effective nonrelativistic interactions between the dark matter and nuclei. There was another work in which the dark matter couples only to the top quark and corresponding predictions at direct and indirect detection experiments as well as colliders are obtained [8].

In the present work, we first estimate the lower bounds on the new interactions based on the fact that if a particular interaction is the only contribution that can thermalize the dark matter particle in the early Universe, we require this interaction must be strong enough such that the resulting relic density would not overclose the Universe. In addition, we proceed to calculate the antiproton flux coming from the effective interactions. We expect the latest antiproton data from PAMELA [9] can put a strong constraint on the size of the interactions, based on the fact that the existing data do not allow excessive flux above the conventional background. On the other hand, the positron spectrum from PAMELA [10] showed some excessive above the conventional background and thus if we used it to constrain the model, it in general gives a weaker constraint than the antiproton flux [8, 11]. Therefore, in this work we focus on the constraints from antiproton flux. Similar ideas of using the antiproton flux from earlier PAMELA data was considered in Ref. [12] to confront the low energy CoGeNT experiment [13] for a lower mass DM.

The organization of the paper is as follows. In the next section, we describe the interactions between the dark matter particle and the SM particles, in particular quarks and gluons. In Sec. III, we study the velocity dependence of the effective operators based on 
the nonrelativistic reduction. In Sec. IV, we calculate the annihilation rates during the freeze-out and make sure that the interactions would not overclose the Universe. In Sec. V, we calculate the antiproton spectrum due to the dark matter annihilation in Galactic Halo. We compare with other constraints and conclude in Sec. VI.

\section{EFFECTIVE INTERACTIONS}

Let us start by assuming the dark matter is a Dirac fermion and its effective interactions with light quarks via a (axial) vector-boson or tensor-type exchange are given by the following dimension 6 operators

$$
\mathcal{L}_{i=1-6}=\frac{C}{\Lambda_{i}^{2}}\left(\bar{\chi} \Gamma_{1} \chi\right)\left(\bar{q} \Gamma_{2} q\right)
$$

where $\Gamma_{1,2}=\gamma^{\mu}, \gamma^{\mu} \gamma^{5}, \sigma^{\mu \nu}$ or $\sigma^{\mu \nu} \gamma^{5}$ with $\sigma^{\mu \nu} \equiv i\left(\gamma^{\mu} \gamma^{\nu}-\gamma^{\nu} \gamma^{\mu}\right) / 2$, and $C$ is an effective coupling constant of order $O(1)$. For Majorana fermion the $\Gamma_{1}=\gamma^{\mu}$ or $\sigma^{\mu \nu}$ type interaction is identically zero, and so for vector or tensor type interaction the fermion $\chi$ in Eq.(2) is understood to be Dirac. Explicitly, we assume the dark matter candidate to be Dirac, but the results are also applicable to Majorana dark matter.

Next set of operators are associated with (pseudo) scalar-boson-type exchange

$$
\mathcal{L}_{i=7-10}=\frac{C m_{q}}{\Lambda_{i}^{3}}\left(\bar{\chi} \Gamma_{1} \chi\right)\left(\bar{q} \Gamma_{2} q\right)
$$

where $\Gamma_{1,2}=1$ or $i \gamma^{5}$. The $m_{q}$ dependence in the coupling strength is explicitly shown for scalar-type interactions. We use the current quark masses in the Lagrangian given by [14]:

$$
\begin{aligned}
& m_{u}=0.0025 \mathrm{GeV}, \quad m_{d}=0.005 \mathrm{GeV}, \quad m_{s}=0.101 \mathrm{GeV} \\
& m_{c}=1.27 \mathrm{GeV}, \quad m_{b}=4.19 \mathrm{GeV}, \quad m_{t}=172 \mathrm{GeV}
\end{aligned}
$$

Another light degree of freedom that couples to the Dirac dark matter is the gluon field ${ }^{1}$

$$
\begin{aligned}
\mathcal{L}_{i=11-12} & =\frac{C \alpha_{s}\left(2 m_{\chi}\right)}{4 \Lambda_{i}^{3}}(\bar{\chi} \Gamma \chi) G^{a \mu \nu} G_{\mu \nu}^{a} \\
\mathcal{L}_{i=13-14} & =\frac{C \alpha_{s}\left(2 m_{\chi}\right)}{4 \Lambda_{i}^{3}}(\bar{\chi} \Gamma \chi) G^{a \mu \nu} \tilde{G}_{\mu \nu}^{a}
\end{aligned}
$$

\footnotetext{
${ }^{1}$ We do not study the other gauge bosons, like $W$ and $Z$ bosons, because they decay into light quarks which then fragment into $\bar{p}$. The secondary antiproton spectrum would be softer in this case.
} 
where $\Gamma=1$ or $i \gamma^{5}$ and the strong coupling constant is evaluated at the scale $2 m_{\chi}$ where $m_{\chi}$ is the dark matter mass.

Finally, we also write down the corresponding operators for complex scalar dark matter. Again, we note that the interactions for real scalar dark matter is similar to complex one and differ by a factor of two. We simply focus on the complex scalar dark matter. The operators corresponding to vector boson exchange are

$$
\mathcal{L}_{i=15,16}=\frac{C}{\Lambda_{i}^{2}}\left(\chi^{\dagger} \overleftrightarrow{\partial_{\mu}} \chi\right)\left(\bar{q} \gamma^{\mu} \Gamma q\right)
$$

where $\Gamma=1$ or $\gamma^{5}$ and $\chi^{\dagger} \overleftrightarrow{\partial_{\mu}} \chi=\chi^{\dagger}\left(\partial_{\mu} \chi\right)-\left(\partial_{\mu} \chi^{\dagger}\right) \chi$. Those corresponding to a scalar boson exchange are

$$
\mathcal{L}_{i=17,18}=\frac{C m_{q}}{\Lambda_{i}^{2}}\left(\chi^{\dagger} \chi\right)(\bar{q} \Gamma q),
$$

where $\Gamma=1$ or $i \gamma^{5}$. The corresponding gluonic operators are

$$
\begin{aligned}
\mathcal{L}_{i=19} & =\frac{C \alpha_{s}\left(2 m_{\chi}\right)}{4 \Lambda_{i}^{3}}\left(\chi^{\dagger} \chi\right) G^{a \mu \nu} G_{a \mu \nu}, \\
\mathcal{L}_{i=20} & =\frac{i C \alpha_{s}\left(2 m_{\chi}\right)}{4 \Lambda_{i}^{3}}\left(\chi^{\dagger} \chi\right) G^{a \mu \nu} \tilde{G}_{a \mu \nu} .
\end{aligned}
$$

The whole list of operators are listed in Table I. We will consider one operator at a time, and set the coefficient $C=1$ for simplicity.

Note that in calculating the annihilation rate in the freeze-out in the early Universe, we include all light-quark flavors $(u, d, s, c, b)$ as well as the heavy top quark which is relevant when $m_{\chi}$ rises above the top quark threshold. However, in the calculation of the antiproton flux from dark matter annihilation in the present Galactic halo, we only include the lightquark flavors. We ignore the $\chi \bar{\chi} \rightarrow t \bar{t}$ contribution, because the $t$ and $\bar{t}$ first decay into $b W \rightarrow b q \bar{q}^{\prime}$ before each light quark undergoes fragmentation into hadrons, including proton and antiproton. Therefore, the antiproton spectrum would be significantly softer than the direct fragmentation as in $\chi \bar{\chi} \rightarrow q \bar{q}[8]$. We anticipate that by ignoring the $t \bar{t}$ contribution the limits we obtain from the PAMELA data would not be affected to any significant amount in the case we just use the five light-quark flavors.

\section{VELOCITY DEPENDENCE IN THE NONRELATIVISTIC LIMITS}

In order to easily understand the results that we obtain in Sec. V, we are going to examine the dependence of the annihilation cross section on the velocity of the dark matter particle 
TABLE I: The list of effective interactions between the dark matter and the light degrees of freedom (quark or gluon). We have suppressed the color index on the quark and gluon fields. These operators have also been analyzed in Refs. [3, 5, 6].

\begin{tabular}{|c|c|}
\hline Operator & Coefficient \\
\hline \multicolumn{2}{|c|}{ Dirac DM, Vector Boson Exchange } \\
\hline$O_{1}=\left(\bar{\chi} \gamma^{\mu} \chi\right)\left(\bar{q} \gamma_{\mu} q\right)$ & $\frac{C}{\Lambda^{2}}$ \\
\hline$O_{2}=\left(\bar{\chi} \gamma^{\mu} \gamma^{5} \chi\right)\left(\bar{q} \gamma_{\mu} q\right)$ & $\frac{C}{\Lambda^{2}}$ \\
\hline$O_{3}=\left(\bar{\chi} \gamma^{\mu} \chi\right)\left(\bar{q} \gamma_{\mu} \gamma^{5} q\right)$ & $\frac{C}{\Lambda^{2}}$ \\
\hline$O_{4}=\left(\bar{\chi} \gamma^{\mu} \gamma^{5} \chi\right)\left(\bar{q} \gamma_{\mu} \gamma^{5} q\right)$ & $\frac{C}{\Lambda^{2}}$ \\
\hline$O_{5}=\left(\bar{\chi} \sigma^{\mu \nu} \chi\right)\left(\bar{q} \sigma_{\mu \nu} q\right)$ & $\frac{C}{\Lambda^{2}}$ \\
\hline$O_{6}=\left(\bar{\chi} \sigma^{\mu \nu} \gamma^{5} \chi\right)\left(\bar{q} \sigma_{\mu \nu} q\right)$ & $\frac{C}{\Lambda^{2}}$ \\
\hline \multicolumn{2}{|c|}{ Dirac DM, Scalar Boson Exchange } \\
\hline$O_{7}=(\bar{\chi} \chi)(\bar{q} q)$ & $\frac{C m_{q}}{\Lambda^{3}}$ \\
\hline$O_{8}=\left(\bar{\chi} \gamma^{5} \chi\right)(\bar{q} q)$ & $\frac{i C m_{q}}{\Lambda^{3}}$ \\
\hline$O_{9}=(\bar{\chi} \chi)\left(\bar{q} \gamma^{5} q\right)$ & $\frac{i C m_{q}}{\Lambda^{3}}$ \\
\hline$O_{10}=\left(\bar{\chi} \gamma^{5} \chi\right)\left(\bar{q} \gamma^{5} q\right)$ & $\frac{C m_{q}}{\Lambda^{3}}$ \\
\hline \multicolumn{2}{|c|}{ Dirac DM, Gluonic } \\
\hline$O_{11}=(\bar{\chi} \chi) G_{\mu \nu} G^{\mu \nu}$ & $\frac{C \alpha_{s}}{4 \Lambda^{3}}$ \\
\hline$O_{12}=\left(\bar{\chi} \gamma^{5} \chi\right) G_{\mu \nu} G^{\mu \nu}$ & $\frac{i C \alpha_{s}}{4 \Lambda^{3}}$ \\
\hline$O_{13}=(\bar{\chi} \chi) G_{\mu \nu} \tilde{G}^{\mu \nu}$ & $\frac{C \alpha_{s}}{4 \Lambda^{3}}$ \\
\hline$\underline{O_{14}}=\left(\bar{\chi} \gamma^{5} \chi\right) G_{\mu \nu} \tilde{G}^{\mu \nu}$ & $\frac{i C \alpha_{s}}{4 \Lambda^{3}}$ \\
\hline \multicolumn{2}{|c|}{ Complex Scalar DM, Vector Boson Exchange } \\
\hline$O_{15}=\left(\chi^{\dagger} \overleftrightarrow{\partial_{\mu}} \chi\right)\left(\bar{q} \gamma^{\mu} q\right)$ & $\frac{C}{\Lambda^{2}}$ \\
\hline$O_{16}=\left(\chi^{\dagger} \overleftrightarrow{\partial_{\mu}} \chi\right)\left(\bar{q} \gamma^{\mu} \gamma^{5} q\right)$ & $\frac{C}{\Lambda^{2}}$ \\
\hline \multicolumn{2}{|c|}{ Complex Scalar DM, Scalar Vector Boson Exchange } \\
\hline$O_{17}=\left(\chi^{\dagger} \chi\right)(\bar{q} q)$ & $\frac{C m_{q}}{\Lambda^{2}}$ \\
\hline$O_{18}=\left(\chi^{\dagger} \chi\right)\left(\bar{q} \gamma^{5} q\right)$ & $\frac{i C m_{q}}{\Lambda^{2}}$ \\
\hline \multicolumn{2}{|c|}{ Complex Scalar DM, Gluonic } \\
\hline$O_{19}=\left(\chi^{\dagger} \chi\right) G_{\mu \nu} G^{\mu \nu}$ & $\frac{C \alpha_{s}}{4 \Lambda^{2}}$ \\
\hline$O_{20}=\left(\chi^{\dagger} \chi\right) G_{\mu \nu} \tilde{G}^{\mu \nu}$ & $\frac{i C \alpha_{s}}{4 \Lambda^{2}}$ \\
\hline
\end{tabular}


in the nonrelativistic limit. The current velocity of the dark matter in the Universe around the Sun is about $v \approx 300 \mathrm{~km} \mathrm{~s}^{-1} \approx 10^{-3} c$, where $c$ is the speed of the light. Given such a small $v$ the dependence on $v$ is very important. For instance, the annihilation rate of the Dirac DM with a scalar boson exchange, given by the interaction in $O_{7}$, would suffer from a factor of $v^{2}$. Therefore, we expect the antiproton flux from such an operator would be very small.

Let us consider the operators $O_{1}$ to $O_{6}$ with (axial) vector-boson/tensor-like exchange. In terms of Dirac spinors $(\psi$ and $\bar{\psi})$ the relevant part of the annihilation amplitude of the Dirac DM is given by

$$
\bar{\psi}\left(p_{2}\right) \Gamma \psi\left(p_{1}\right)
$$

for $\bar{\chi}\left(p_{2}\right) \chi\left(p_{1}\right) \rightarrow q \bar{q}$ and $\Gamma=\gamma^{\mu}, \gamma^{\mu} \gamma^{5}, \sigma^{\mu \nu}$ or $\sigma^{\mu \nu} \gamma^{5}$. In Dirac representation, the gamma matrices are given by

$$
\gamma^{0}=\left(\begin{array}{cc}
I & 0 \\
0 & -I
\end{array}\right), \gamma^{i}=\left(\begin{array}{cc}
0 & \sigma_{i} \\
-\sigma_{i} & 0
\end{array}\right), \gamma^{5}=\left(\begin{array}{cc}
0 & I \\
I & 0
\end{array}\right)
$$

where $\sigma_{i}(i=1,2,3)$ are the Pauli matrices. In the nonrelativistic limit, the spinor for the $\operatorname{DM} \chi$ is $\psi=\xi\left(\begin{array}{l}1 \\ \epsilon\end{array}\right)$, where $\epsilon=O(v / c)$. On the other hand, the spinor for the antiparticle $\bar{\chi}$ is $\bar{\psi}=\eta^{\dagger}(\epsilon, 1) \gamma^{0} .{ }^{2}$ Therefore, we can expand $\bar{\psi} \gamma^{\mu} \psi$, in the nonrelativistic limit, as

$$
\begin{aligned}
\bar{\psi} \gamma^{0} \psi & \simeq 2 \epsilon \eta^{\dagger} \xi \\
\bar{\psi} \gamma^{i} \psi & \simeq\left(1+\epsilon^{2}\right) \eta^{\dagger} \sigma_{i} \xi
\end{aligned}
$$

where the space-like parts are not suppressed by $v / c$. On the other hand, $\bar{\psi} \gamma^{\mu} \gamma^{5} \psi$ in the nonrelativistic limit are

$$
\begin{aligned}
& \bar{\psi} \gamma^{0} \gamma^{5} \psi \simeq\left(1+\epsilon^{2}\right) \eta^{\dagger} \xi \\
& \bar{\psi} \gamma^{i} \gamma^{5} \psi \simeq 2 \epsilon \eta^{\dagger} \sigma_{i} \xi
\end{aligned}
$$

where the space-like parts are suppressed by $v / c$. It is clear that in the nonrelativistic limit the time-like and space-like parts behave very differently. We can then consider them separately when it is squared, traced, and contracted with the trace of the light quark leg. If

\footnotetext{
${ }^{2}$ It is different from the direct scattering with a nucleon, where we need the $\bar{\psi}=\xi^{\dagger}(1, \epsilon) \gamma^{0}$.
} 
we look at the trace of the part $\left(\bar{q} \gamma^{\mu} q\right)$ or $\left(\bar{q} \gamma^{\mu} \gamma^{5} q\right)$ in the annihilation amplitude, the timelike part after being squared and traced gives a quantity close to zero, while the space-like part after squared and traced gives a quantity in the order of $m_{\chi}^{2}$. Therefore, it is clear now that $\bar{\psi} \gamma^{\mu} \psi$ multiplied to $\left(\bar{q} \gamma_{\mu} q\right)$ or $\left(\bar{q} \gamma_{\mu} \gamma^{5} q\right)$ will not be suppressed, while $\bar{\psi} \gamma^{\mu} \gamma^{5} \psi$ multiplied to $\left(\bar{q} \gamma_{\mu} q\right)$ or $\left(\bar{q} \gamma_{\mu} \gamma^{5} q\right)$ will be suppressed. The above observation is consistent with the results that we obtain in Sec. V. From Table II the limits on $O_{1}$ and $O_{3}$ are much stronger than those on $\mathrm{O}_{2}$ and $\mathrm{O}_{4}$. The operators $\mathrm{O}_{5}$ and $O_{6}$ contain unsuppressed components in $\mu \nu=0 i$ entries.

In contrast, the operators $O_{7}$ to $O_{10}$ with (pseudo) scalar-boson exchange are suppressed when there is no $\gamma^{5}$ in the fermion line of $\chi$, which is obvious from the following in the nonrelativistic limit

$$
\begin{aligned}
\bar{\psi} \psi & \sim \epsilon \eta^{\dagger} \xi \\
\bar{\psi} \gamma^{5} \psi & \sim \eta^{\dagger} \xi
\end{aligned}
$$

Again, it is then obvious from Table II that the limits on $O_{8}$ and $O_{10}$ are much stronger than those on $O_{7}$ and $O_{9}$. The gluonic operators in $O_{11-14}$ follow similar patterns: $O_{11,13}$ are suppressed relative to $O_{12,14}$.

It is also straightforward to understand the velocity dependence for the scalar DM, represented by the operators $O_{15-20}$. Except for $O_{15,16}$ all of them are not suppressed by $v / c$, because of the presence of the $\overleftrightarrow{\partial_{\mu}}$ in $O_{15,16}$. This $\partial_{\mu}$ will bring down $p_{\mu}$ in the vertex factor. While $p_{0}$ is of order $m_{\chi}, p_{i}$ is $v / c$. Therefore, when it contracts with the quark leg, the overall result is suppressed by $v / c$.

\section{ANNIHILATION RATES AROUND THE FREEZE-OUT}

It is obvious from Eq. (1) that if the annihilation rate falls below $1 \mathrm{pb}$, then the thermal relic density would be more than the WMAP data can allow. Therefore, we have to restrict the annihilation rate to be larger than about $1 \mathrm{pb}$. More precisely, using the most recent WMAP result on dark matter density $\Omega_{\mathrm{CDM}} h^{2}=0.1099 \pm 0.0062$ [1] the annihilation rate is

$$
\langle\sigma v\rangle \simeq 0.91 \mathrm{pb}
$$

We assume $v \approx 0.3$ at around the freeze-out time in the early Universe. 
We calculate the annihilation rates for all the operators and show the contours in Fig. 1, Fig. 2, and Fig. 3 for Dirac DM with (axial) vector-boson/tensor-like exchanges, Dirac DM with (pseudo) scalar-boson exchanges and Dirac DM with gluonic interactions, and scalar DM, respectively. The solid lines are the contours in $\left(m_{\chi}, \Lambda\right)$ plane with annihilation rate $\sigma v=0.91 \mathrm{pb}$. All the values of $\Lambda$ above the solid lines would give a too small annihilation rate, and thus would result in a too large thermal relic density. Therefore, the $\Lambda$ below the solid lines is the allowed region. The cusp structures in the plots are due to the onset of the top quark contributions when $m_{\chi}>m_{t}$. The dashed lines are the limits from the antiproton flux, which will be explained in the next section.

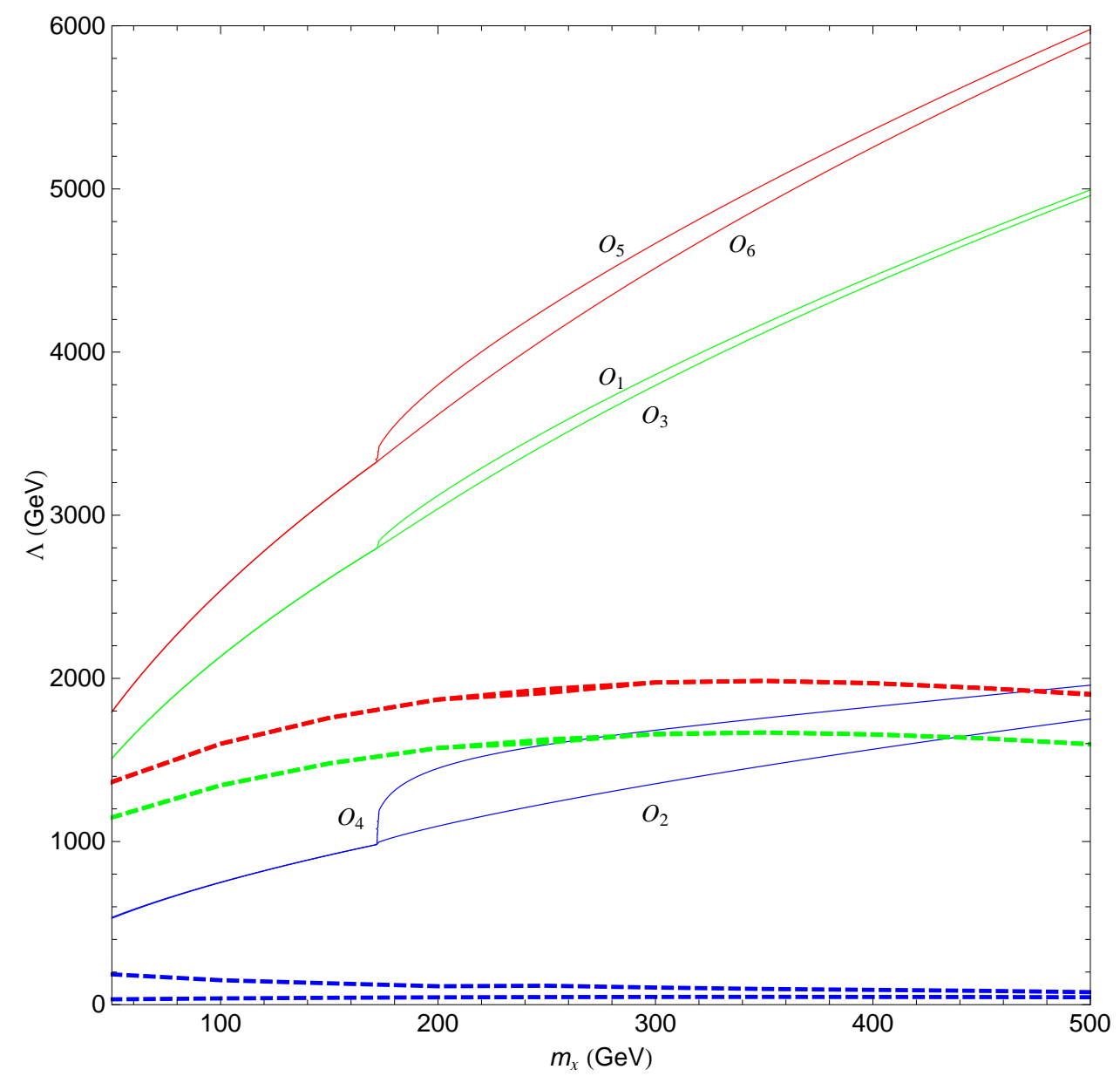

FIG. 1: The upper limits on $\Lambda_{i}$ due to the relic density constraint, requiring $\sigma v \geq 0.91 \mathrm{pb}$ for operators $O_{1-6}$ involving Dirac DM with (axial) vector-boson/tensor-like exchanges (shown by solid lines). The lower limits on $\Lambda_{i}$ due to the antiproton-flux constraint at $3 \sigma$ level for the same operators (shown by dashed lines with the corresponding color). 


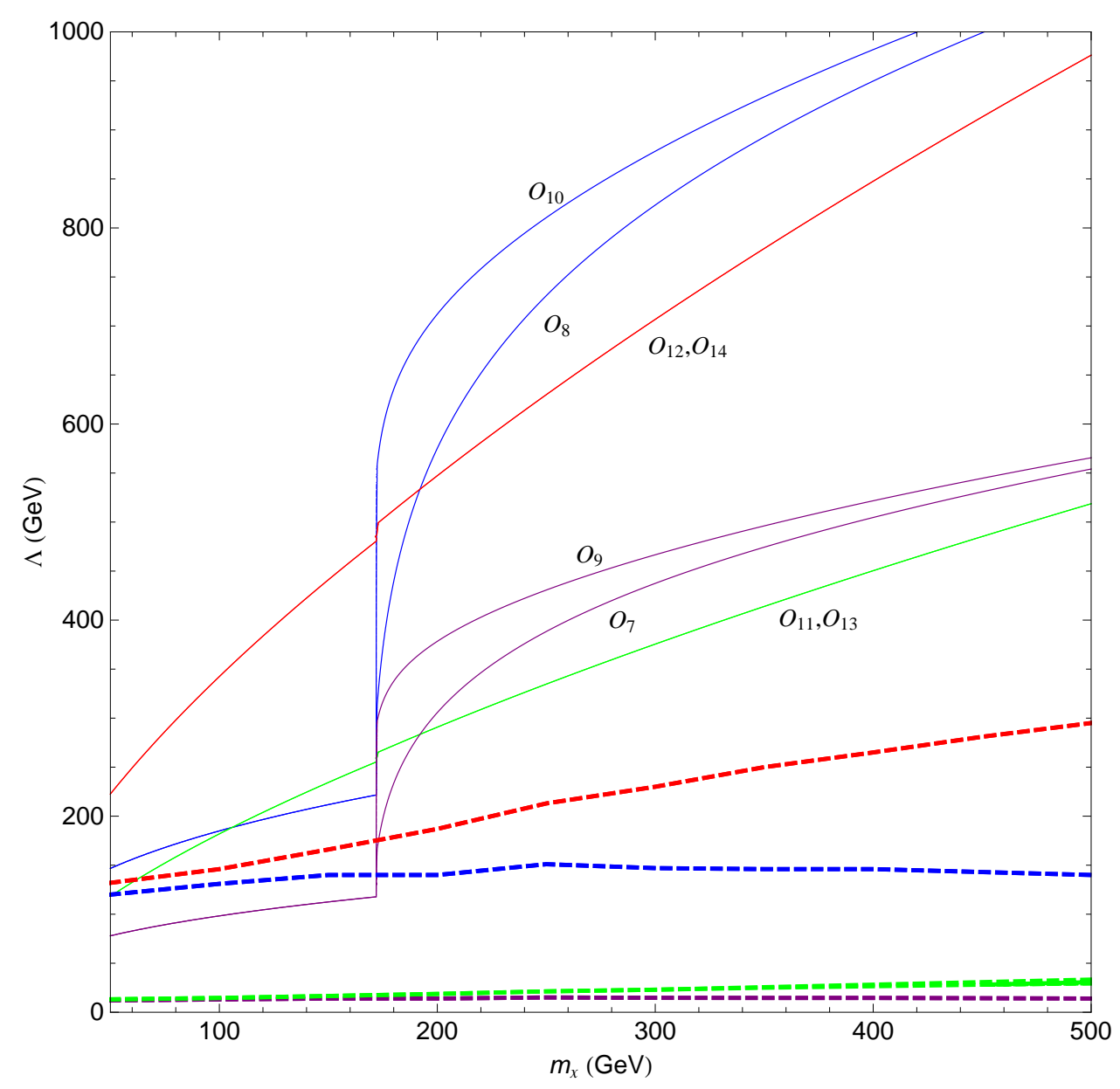

FIG. 2: The same as Fig. 1, but for operators $O_{7-14}$ involving Dirac DM with (pseudo) scalar-boson exchanges $\left(O_{7-10}\right)$ and Dirac DM with gluonic interactions $\left(O_{11-14}\right)$.

\section{ANTIPROTON FLUX}

An important method to detect the dark matter is by measuring its annihilation products in Galactic halo. Current experiments can detect the positron, antiproton, gamma ray, and deuterium from dark matter annihilation. The Milky Way halo may contain clumps of dark matter, from where the annihilation of dark matter particles may give rise to large enough signals, such as positron and antiproton, that can be identified by a number of antimatter search experiments. The most recent ones come from PAMELA [9, 10], which showed a spectacular rise in the positron spectrum but an expected spectrum for antiproton. It may be due to nearby pulsars or dark matter annihilation or decays. If it is really due to dark matter annihilation, the dark matter would have very strange properties, because it only gives positrons in the final products but not antiproton. Here we adopt a conservative 


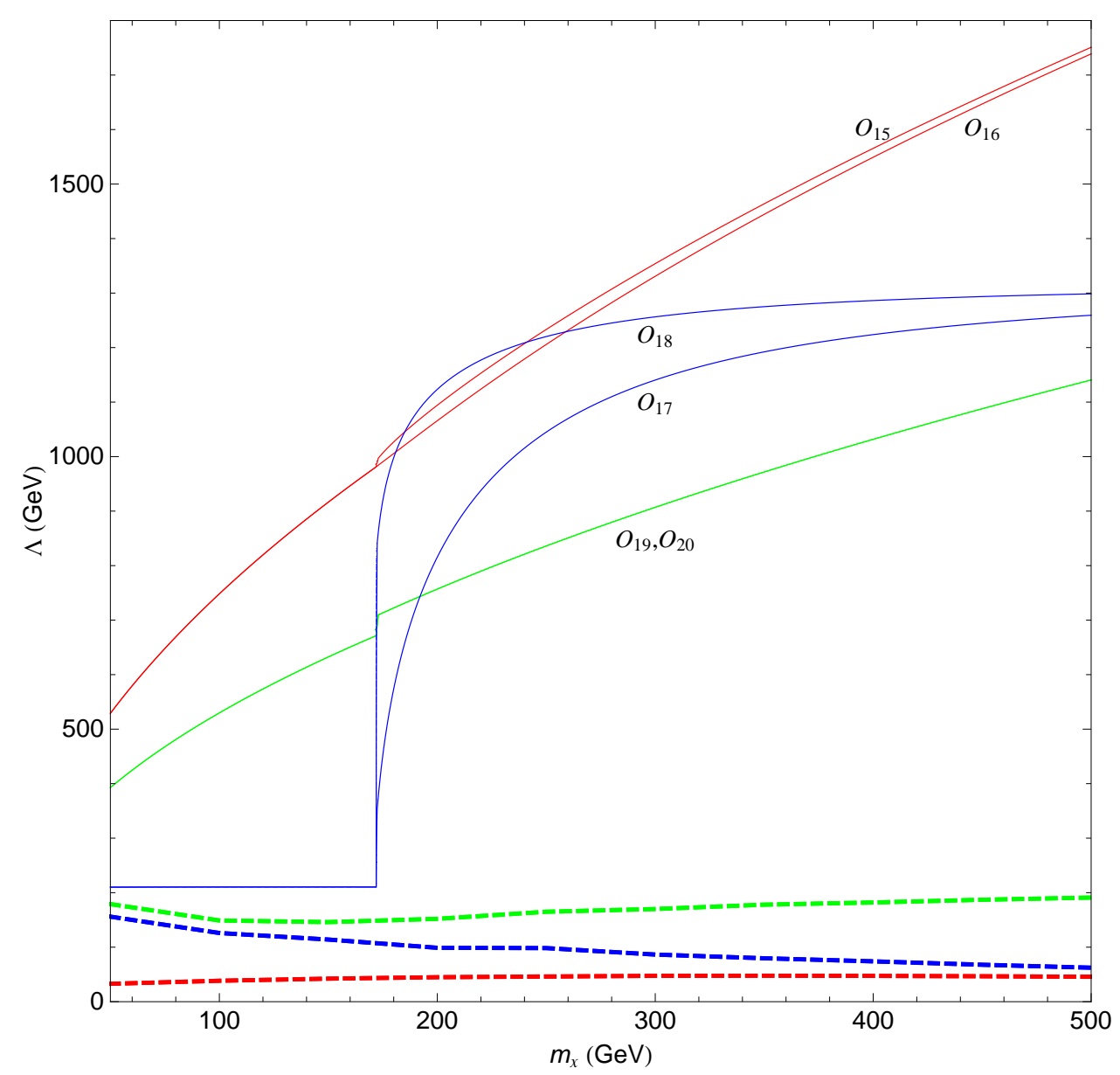

FIG. 3: The same as Fig. 1, but for operators $O_{15-20}$ involving complex scalar DM.

approach. We use the observed antiproton as a constraint on the annihilation products in $\chi \bar{\chi}$ annihilation. In general, the positron data would give a weaker constraint as it allows some level of signals of dark matter annihilation [8].

The antiproton flux can be obtained by solving the diffusion equation with the corresponding diffusion terms and the appropriate source term for the input antiproton spectrum:

$$
Q_{\mathrm{ann}}=\eta\left(\frac{\rho_{\mathrm{CDM}}}{M_{\mathrm{CDM}}}\right)^{2} \sum\langle\sigma v\rangle_{\bar{p}} \frac{d N_{\bar{p}}}{d T_{\bar{p}}}
$$

where $\eta=1 / 2(1 / 4)$ for (non-)identical initial state, and $T_{\bar{p}}$ is the kinetic energy of the antiproton which is conventionally used instead of the total energy. We again solve the diffusion equation using GALPROP [15].

In our case, the dominant contribution comes from

$$
\chi \bar{\chi} \rightarrow q \bar{q} \rightarrow \bar{p}+X
$$




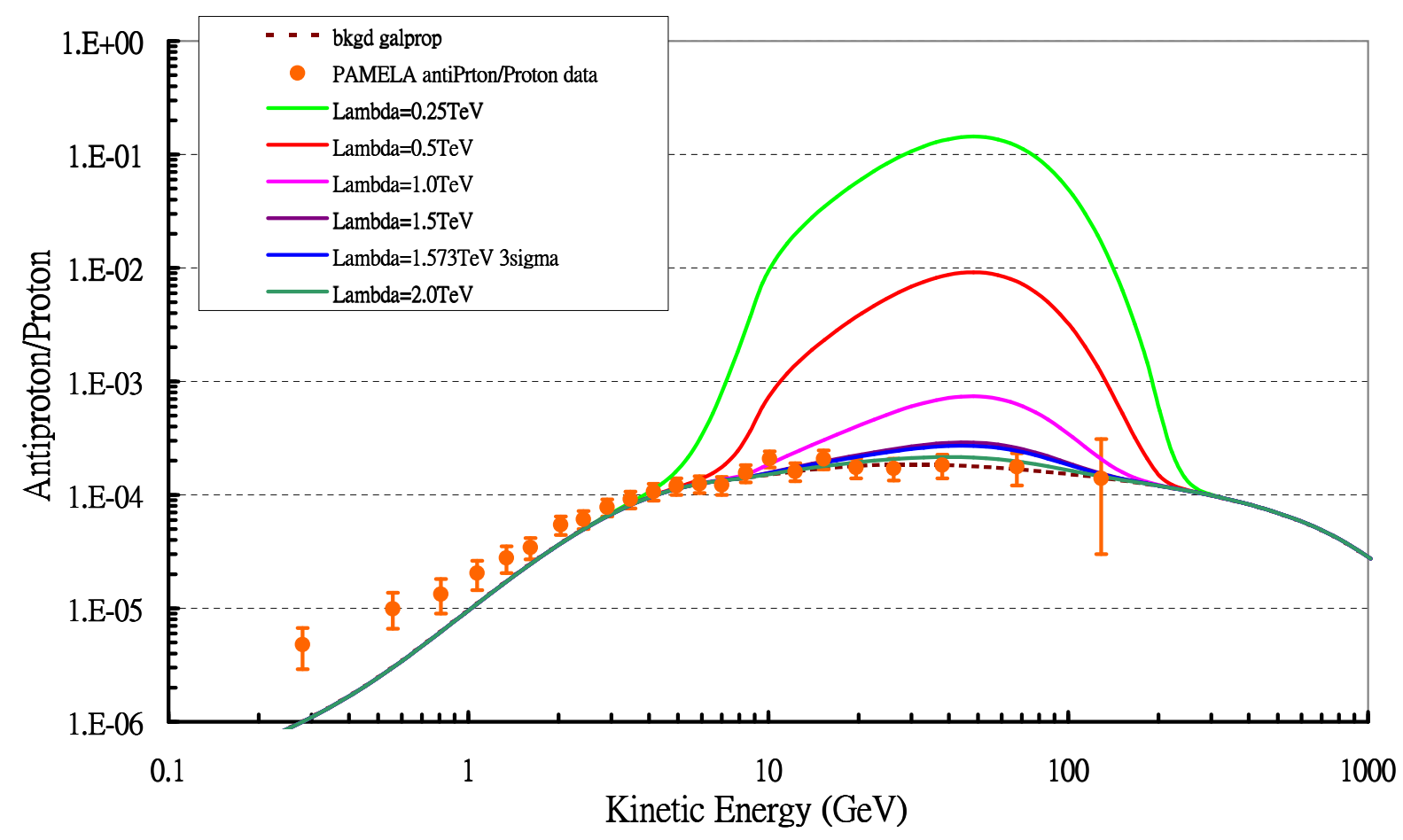

FIG. 4: Antiproton fraction spectrum predicted for the operator $O_{1}=\frac{1}{\Lambda^{2}}\left(\bar{\chi} \gamma^{\mu} \chi\right)\left(\bar{q} \gamma_{\mu} q\right)$ for a few values of $\Lambda$. The mass of the dark matter is chosen to be $200 \mathrm{GeV}$ here. The data points are from PAMELA [9].

in which all the $q, \bar{q}(q=u, d, c, s, b)$ have probabilities fragmenting into $\bar{p}$. We adopt a publicly available code [16] to calculate the fragmentation function $D_{q \rightarrow h}(z)$ for any quark $q$ into hadrons $h$, e.g., $p, \bar{p}, \pi$. The fragmentation function is then convoluted with energy spectrum $d N / d T$ of the light quark to obtain the energy spectrum of the antiproton $d N / d T_{\bar{p}}$. The source term $d N / d T_{\bar{p}}$ is then implemented into GALPROP to calculate the propagation from the halo to the Earth. We display the energy spectrum for the antiproton fraction in Fig. 4 for the operator $O_{1}$, in which various values of $\Lambda$ are chosen. We only chose a typical operator. The effects of other operators are similar.

Here we adopt a simple statistical measure to quantify the effect of each operator. We 
calculate the $3 \sigma$ limit on each scale $\Lambda_{i}$. We assume the data agree well with the expected background, and then we calculate the $\chi^{2}$ with finite $\Lambda_{i}$ 's until we obtain a $\chi^{2}$ difference of $\chi^{2}-\chi_{\mathrm{bkgd}}^{2}=9(3 \sigma)$. Note that the uncertainties in the background estimation of the low energy range $(\lesssim 4 \mathrm{GeV})$ are large in GALPROP, mainly because of different profiles employed. We therefore focus on the data points above $4 \mathrm{GeV}$ when we calculate the $\chi^{2}$. The data points above $4 \mathrm{GeV}$ enjoy a small $\chi^{2}=5.0$ for 13 degrees of freedom. We tabulate all the lower limits of $\Lambda_{i} \mathrm{~s}$ in Table $\amalg$ for $m_{\chi}=50,100,200$ and $400 \mathrm{GeV}$.

Limits for intermediate values of $m_{\chi}$ are shown in Fig. 1, Fig. 2, and Fig. 3 for Dirac DM with (axial) vector-boson/tensor-like exchanges, Dirac DM with (pseudo) scalar-boson exchanges and Dirac DM with gluonic interactions, and scalar DM, respectively. The solid lines are the upper limits due to thermal relic density. The dashed lines are the lower limits due to antiproton flux. Therefore, for each operator there is a valid range of $\Lambda$. For example, the operator $O_{1,3}$ requires $1.6 \mathrm{TeV} \lesssim \Lambda_{1,3} \lesssim 3 \mathrm{TeV}$ for $m_{\chi}=200 \mathrm{GeV}$. The best limit is obtained for the Dirac DM with tensor interactions in $O_{5,6}$ at $1.9 \mathrm{TeV} \lesssim \Lambda_{5,6} \lesssim 3.6 \mathrm{TeV}$ for $m_{\chi}=200 \mathrm{GeV}$. In general, the Dirac DM with vector-like exchanges gives the best limit, except for the operators $O_{2,4}$, which are well known for velocity suppression. The operators $O_{7-10}$ for Dirac DM with scalar-boson exchanges naturally give rather weak limits, because of the factor $m_{q}$ in the coupling constant. In addition, operators $O_{7,9}$ are suppressed by the velocity. The gluonic interactions in the operators $O_{11-14}$ also give mild limits because of the $\alpha_{s} \approx 10^{-1}$ in the coupling constant, in which the operators $O_{11,13}$ are further suppressed by velocity. On the other hand, the operators for scalar DM give rather mild limits, especially $O_{15,16}$ give the weakest limits, because the derivative couplings in $O_{15,16}$ bring down a factor of momentum.

\section{DISCUSSION AND CONCLUSIONS}

Here we do a comparison with the limits obtained in Ref. [6], in which limits from relic density, Tevatron, and gamma-ray are shown. Comparisons are summarized as follows.

1. The limits due to relic density obtained in this work are consistent with results of Ref. [6].

2. In Fig. 5 of Ref. [6], the limits for their $D_{1-4}$ (corresponding to our $O_{7-10}$, Dirac DM 
TABLE II: The $3 \sigma$ lower limits on the operators listed in Table I. We take the coefficient $C=1$ with $m_{\chi}=50,100,200$ and $400 \mathrm{GeV}$. We have used the PAMELA data points above the kinetic energy $T=4 \mathrm{GeV}$ in our analysis, because of the large uncertainty of the theoretical background at low energy. The $\chi^{2}(\mathrm{bkdg})=5.0$.

\begin{tabular}{|c|c|c|c|c|}
\hline \multirow[t]{2}{*}{ Operators } & \multicolumn{4}{|l|}{$\Lambda(\mathrm{TeV})$} \\
\hline & $m_{\chi}(\mathrm{GeV})=50$ & 100 & 200 & 400 \\
\hline \multicolumn{5}{|c|}{ Dirac DM, Vector Boson Exchange } \\
\hline$O_{1}=\left(\bar{\chi} \gamma^{\mu} \chi\right)\left(\bar{q} \gamma_{\mu} q\right)$ & 1.15 & 1.34 & 1.57 & 1.66 \\
\hline$O_{2}=\left(\bar{\chi} \gamma^{\mu} \gamma^{5} \chi\right)\left(\bar{q} \gamma_{\mu} q\right)$ & 0.033 & 0.038 & 0.045 & 0.047 \\
\hline$O_{3}=\left(\bar{\chi} \gamma^{\mu} \chi\right)\left(\bar{q} \gamma_{\mu} \gamma^{5} q\right)$ & 1.15 & 1.34 & 1.57 & 1.66 \\
\hline$O_{4}=\left(\bar{\chi} \gamma^{\mu} \gamma^{5} \chi\right)\left(\bar{q} \gamma_{\mu} \gamma^{5} q\right)$ & 0.19 & 0.15 & 0.11 & 0.09 \\
\hline$O_{5}=\left(\bar{\chi} \sigma^{\mu \nu} \chi\right)\left(\bar{q} \sigma_{\mu \nu} q\right)$ & 1.37 & 1.60 & 1.87 & 1.97 \\
\hline$O_{6}=\left(\bar{\chi} \sigma^{\mu \nu} \gamma^{5} \chi\right)\left(\bar{q} \sigma_{\mu \nu} q\right)$ & 1.36 & 1.60 & 1.87 & 1.97 \\
\hline \multicolumn{5}{|c|}{ Dirac DM, Scalar Boson Exchange } \\
\hline$O_{7}=(\bar{\chi} \chi)(\bar{q} q)$ & 0.012 & 0.013 & 0.014 & 0.015 \\
\hline$O_{8}=\left(\bar{\chi} \gamma^{5} \chi\right)(\bar{q} q)$ & 0.12 & 0.13 & 0.14 & 0.15 \\
\hline$O_{9}=(\bar{\chi} \chi)\left(\bar{q} \gamma^{5} q\right)$ & 0.012 & 0.013 & 0.014 & 0.015 \\
\hline$O_{10}=\left(\bar{\chi} \gamma^{5} \chi\right)\left(\bar{q} \gamma^{5} q\right)$ & 0.12 & 0.13 & 0.14 & 0.15 \\
\hline \multicolumn{5}{|c|}{ Dirac DM, Gluonic } \\
\hline$O_{11}=(\bar{\chi} \chi) G_{\mu \nu} G^{\mu \nu}$ & 0.013 & 0.015 & 0.019 & 0.027 \\
\hline$O_{12}=\left(\bar{\chi} \gamma^{5} \chi\right) G_{\mu \nu} G^{\mu \nu}$ & 0.13 & 0.15 & 0.19 & 0.27 \\
\hline$O_{13}=(\bar{\chi} \chi) G_{\mu \nu} \tilde{G}^{\mu \nu}$ & 0.013 & 0.015 & 0.019 & 0.027 \\
\hline$O_{14}=\left(\bar{\chi} \gamma^{5} \chi\right) G_{\mu \nu} \tilde{G}^{\mu \nu}$ & 0.13 & 0.15 & 0.19 & 0.27 \\
\hline
\end{tabular}

Complex Scalar DM, Vector Boson Exchange

\begin{tabular}{ll}
\hline$O_{15}=\left(\chi^{\dagger} \overleftrightarrow{\partial_{\mu}} \chi\right)\left(\bar{q} \gamma^{\mu} q\right)$ & 0.0330 .0380 .0450 .047 \\
$O_{16}=\left(\chi^{\dagger} \overleftrightarrow{\partial_{\mu}} \chi\right)\left(\bar{q} \gamma^{\mu} \gamma^{5} q\right)$ & 0.0330 .0380 .0450 .047 \\
\hline
\end{tabular}

Complex Scalar DM, Scalar Vector Boson Exchange

\begin{tabular}{|c|c|c|}
\hline$O_{17}=\left(\chi^{\dagger} \chi\right)(\bar{q} q)$ & 0.16 & $\begin{array}{lll}0.13 & 0.099 & 0.074\end{array}$ \\
\hline$O_{18}=\left(\chi^{\dagger} \chi\right)\left(\bar{q} \gamma^{5} q\right)$ & 0.16 & $\begin{array}{lll}0.13 & 0.099 & 0.074\end{array}$ \\
\hline
\end{tabular}

Complex Scalar DM, Gluonic

\begin{tabular}{|c|c|c|c|c|c|}
\hline$O_{19}=\left(\chi^{\dagger} \chi\right) G_{\mu \nu} G^{\mu \nu}$ & & 0.18 & 0.15 & 0.15 & 0.18 \\
\hline$O_{20}=\left(\chi^{\dagger} \chi\right) G_{\mu \nu} \tilde{G}^{\mu \nu}$ & & 0.18 & 0.15 & 0.15 & 0.18 \\
\hline
\end{tabular}


with (pseudo) scalar-boson exchanges) are shown. The limits obtained from FERMI gamma-ray are about the same as what we obtained from PAMELA antiproton data. The limits from FERMI improve with increasing $m_{\chi}$ while it is almost flat in our case (see Fig. 2).

3. In Fig. 6 of Ref. [6], the limits for their $D_{5-8}$ (corresponding to our $O_{1-4}$, Dirac DM with (axial) vector-boson exchanges) are shown. The limits on $O_{1,3}$ obtained from FERMI gamma-ray are about $0.1-0.5 \mathrm{TeV}$ while the limits that we obtained from antiproton data are $1.1-1.7 \mathrm{TeV}$, significantly stronger. The limits on $O_{2,4}$ are velocity suppressed and are $0.03-0.2 \mathrm{TeV}$.

4. In Fig. 8 of Ref. [6], the limits for their $C_{1,2}$ (corresponding to our $O_{17,18}$, complex scalar DM with scalar-boson exchanges) are shown. The limits that we obtained from antiproton data are slightly stronger than those from FERMI gamma-ray data.

5. In Fig. 9 of Ref. [6], the limits for their $C_{3,4}$ (corresponding to our $O_{15,16}$, complex scalar DM with vector-boson exchanges) are shown. The limits that we obtained from antiproton data are weaker than those from FERMI gamma-ray data.

6. The limits from Tevatron data [6] are rather insensitive to $m_{\chi}$, except when $m_{\chi} \gtrsim$ $200 \mathrm{GeV}$. The limits obtained from FERMI gamma-ray data roughly improve with increasing $m_{\chi}[6]$. The limits obtained from PAMELA antiproton data are in general quite flat.

In summary, we have used an effective interaction approach to investigate the effects of dark matter interactions with light quarks on antiproton flux from the Galactic halo. We have assumed a standard halo density and used the GALPROP to calculate the diffusion. The obtained antiproton flux is then compared with the PAMELA data. We have successfully used the data to obtain a $3 \sigma$ limits on the scale $\Lambda_{i}$. The best limits are from the Dirac DM with vector-boson or tensor-like exchanges. The limits are about $1-2 \mathrm{TeV}$. While the other operators give milder limits. Note that these limits from antiproton flux are lower limits on $\Lambda_{i}$. With the requirement of not exceeding the relic density of the cold dark matter deduced from the WMAP, we also obtain the upper limits on $\Lambda_{i}$. Therefore, both the relic density and antiproton constraints give a valid range for each $\Lambda_{i}$, e.g, $1.6 \mathrm{TeV} \lesssim \Lambda_{1} \lesssim 3 \mathrm{TeV}$ for $m_{\chi}=200 \mathrm{GeV}$. This is a very useful piece of information on the effective interactions of 
dark matter with the SM light quarks that can give useful implications for collider searches and direct detection. 


\section{Appendix A: Annihilation Cross Sections}

Here we list all the formulas for annihilation cross sections of the operators $O_{1}$ to $O_{20}$.

$$
\begin{aligned}
& \frac{d \sigma_{1}}{d z}=\frac{1}{\Lambda^{4}} \frac{N_{C}}{16 \pi s} \frac{\beta_{q}}{\beta_{\chi}}\left[u_{m}^{2}+t_{m}^{2}+2 s\left(m_{\chi}^{2}+m_{q}^{2}\right)\right], \\
& \frac{d \sigma_{2}}{d z}=\frac{1}{\Lambda^{4}} \frac{N_{C}}{16 \pi s} \frac{\beta_{q}}{\beta_{\chi}}\left[u_{m}^{2}+t_{m}^{2}+2 s\left(m_{q}^{2}-m_{\chi}^{2}\right)-8 m_{q}^{2} m_{\chi}^{2}\right], \\
& \frac{d \sigma_{3}}{d z}=\frac{1}{\Lambda^{4}} \frac{N_{C}}{16 \pi s} \frac{\beta_{q}}{\beta_{\chi}}\left[u_{m}^{2}+t_{m}^{2}+2 s\left(m_{\chi}^{2}-m_{q}^{2}\right)-8 m_{q}^{2} m_{\chi}^{2}\right], \\
& \frac{d \sigma_{4}}{d z}=\frac{1}{\Lambda^{4}} \frac{N_{C}}{16 \pi s} \frac{\beta_{q}}{\beta_{\chi}}\left[u_{m}^{2}+t_{m}^{2}-2 s\left(m_{\chi}^{2}+m_{q}^{2}\right)+16 m_{q}^{2} m_{\chi}^{2}\right], \\
& \frac{d \sigma_{5}}{d z}=\frac{1}{\Lambda^{4}} \frac{N_{C}}{4 \pi s} \frac{\beta_{q}}{\beta_{\chi}}\left[2\left(u_{m}^{2}+t_{m}^{2}\right)+2 s\left(m_{\chi}^{2}+m_{q}^{2}\right)+8 m_{q}^{2} m_{\chi}^{2}-s^{2}\right] \text {, } \\
& \frac{d \sigma_{6}}{d z}=\frac{1}{\Lambda^{4}} \frac{N_{C}}{4 \pi s} \frac{\beta_{q}}{\beta_{\chi}}\left[2\left(u_{m}^{2}+t_{m}^{2}\right)+2 s\left(m_{\chi}^{2}+m_{q}^{2}\right)-16 m_{q}^{2} m_{\chi}^{2}-s^{2}\right], \\
& \frac{d \sigma_{7}}{d z}=\frac{m_{q}^{2}}{\Lambda^{6}} \frac{N_{C}}{32 \pi} s \beta_{\chi} \beta_{q}^{3}, \\
& \frac{d \sigma_{8}}{d z}=\frac{m_{q}^{2}}{\Lambda^{6}} \frac{N_{C}}{32 \pi} \frac{s \beta_{q}^{3}}{\beta_{\chi}} \\
& \frac{d \sigma_{9}}{d z}=\frac{m_{q}^{2}}{\Lambda^{6}} \frac{N_{C}}{32 \pi} s \beta_{\chi} \beta_{q}, \\
& \frac{d \sigma_{10}}{d z}=\frac{m_{q}^{2}}{\Lambda^{6}} \frac{N_{C}}{32 \pi} \frac{s \beta_{q}}{\beta_{\chi}} \\
& \frac{d \sigma_{11}}{d z}=\frac{\alpha_{s}^{2}}{\Lambda^{6}} \frac{1}{32 \pi} s^{2} \beta_{\chi}, \\
& \frac{d \sigma_{12}}{d z}=\frac{\alpha_{s}^{2}}{\Lambda^{6}} \frac{1}{32 \pi} \frac{s^{2}}{\beta_{\chi}}, \\
& \frac{d \sigma_{13}}{d z}=\frac{d \sigma_{11}}{d z} \\
& \frac{d \sigma_{14}}{d z}=\frac{d \sigma_{12}}{d z}, \\
& \frac{d \sigma_{15}}{d z}=\frac{1}{\Lambda^{4}} \frac{N_{C}}{4 \pi s} \frac{\beta_{q}}{\beta_{\chi}}\left(u t-m_{q}^{2}(u+t)-m_{\chi}^{4}+m_{q}^{4}\right) \text {, } \\
& \frac{d \sigma_{16}}{d z}=\frac{1}{\Lambda^{4}} \frac{N_{C}}{4 \pi s} \frac{\beta_{q}}{\beta_{\chi}}\left(u t-\left(m_{\chi}^{2}-m_{q}^{2}\right)^{2}\right), \\
& \frac{d \sigma_{17}}{d z}=\frac{m_{q}^{2}}{\Lambda^{4}} \frac{N_{C}}{16 \pi} \frac{\beta_{q}^{3}}{\beta_{\chi}}, \\
& \frac{d \sigma_{18}}{d z}=\frac{m_{q}^{2}}{\Lambda^{4}} \frac{N_{C}}{16 \pi} \frac{\beta_{q}}{\beta_{\chi}}, \\
& \frac{d \sigma_{19}}{d z}=\frac{\alpha_{s}^{2}}{\Lambda^{4}} \frac{1}{16 \pi} \frac{s}{\beta_{\chi}}, \\
& \frac{d \sigma_{20}}{d z}=\frac{d \sigma_{19}}{d z},
\end{aligned}
$$


where $s$ is the square of the center-of-mass energy, $z$ is the cosine of scattering angle, $u_{m}=$ $u-m_{\chi}^{2}-m_{q}^{2}, t_{m}=t-m_{\chi}^{2}-m_{q}^{2}, \beta_{\chi}=\left(1-4 m_{\chi}^{2} / s\right)^{1 / 2}, \beta_{q}=\left(1-4 m_{q}^{2} / s\right)^{1 / 2}$, and $N_{C}$ is the color factor (3 for quarks). We have set the coefficient $C=1$ in these formulas. The annihilation rate in the nonrelativistic limit will then be given by $\sigma \cdot\left(2 \beta_{\chi}\right)$.

\section{Acknowledgments}

The work was supported in parts by the National Science Council of Taiwan under Grant Nos. 99-2112-M-007-005-MY3, and 98-2112-M-001-014-MY3, the NCTS, and the WCU program through the KOSEF funded by the MEST (R31-2008-000-10057-0).

[1] J. Dunkley et al. [WMAP Collaboration], "Five-Year Wilkinson Microwave Anisotropy Probe (WMAP) Observations: Likelihoods and Parameters from the WMAP data," Astrophys. J. Suppl. 180, 306 (2009). [arXiv:0803.0586 [astro-ph]].

[2] G. Bertone, D. Hooper, and J. Silk, "Particle dark matter: Evidence, candidates and constraints," Phys. Rept. 405, 279 (2005) [arXiv:hep-ph/0404175].

[3] Q. H. Cao, C. R. Chen, C. S. Li and H. Zhang, "Effective Dark Matter Model: Relic density, CDMS II, Fermi LAT and LHC," arXiv:0912.4511 [hep-ph].

[4] Y. Bai, P. J. Fox and R. Harnik, "The Tevatron at the Frontier of Dark Matter Direct Detection," arXiv:1005.3797 [hep-ph].

[5] J. Goodman, M. Ibe, A. Rajaraman, W. Shepherd, T. M. P. Tait and H. B. P. Yu, "Constraints on Light Majorana Dark Matter from Colliders," arXiv:1005.1286 [hep-ph]; J. Goodman, M. Ibe, A. Rajaraman, W. Shepherd, T. M. P. Tait and H. B. P. Yu, "Constraints on Dark Matter from Colliders," arXiv:1008.1783 [hep-ph].

[6] J. Goodman, M. Ibe, A. Rajaraman, W. Shepherd, T. M. P. Tait and H. B. P. Yu, "Gamma Ray Line Constraints on Effective Theories of Dark Matter," arXiv:1009.0008 [hep-ph].

[7] J. Fan, M. Reece and L. T. Wang, "Non-relativistic effective theory of dark matter direct detection," arXiv:1008.1591 [hep-ph].

[8] K. Cheung, K. Mawatari, E. Senaha, P. Y. Tseng and T. C. Yuan, "The Top Window for Dark Matter," JHEP 1010, 081 (2010) [arXiv:1009.0618 [hep-ph]]. 
[9] O. Adriani et al. [PAMELA Collaboration], "PAMELA results on the cosmic-ray antiproton flux from $60 \mathrm{MeV}$ to $180 \mathrm{GeV}$ in kinetic energy," Phys. Rev. Lett. 105, 121101 (2010) arXiv:1007.0821 [astro-ph.HE]].

[10] O. Adriani et al. [PAMELA Collaboration], "An anomalous positron abundance in cosmic rays with energies 1.5.100 GeV," Nature 458, 607 (2009). [arXiv:0810.4995 [astro-ph]].

[11] K. Cheung, J. Song and P. Y. Tseng, "Cosmic positron and antiproton constraints on the gauge-Higgs Dark Matter,” JCAP 1009, 023 (2010) [arXiv:1007.0282 [hep-ph]].

[12] I. Low, W. Y. Keung and G. Shaughnessy, "When CoGeNT met PAMELA," arXiv:1010.1774 [hep-ph].

[13] C. E. Aalseth et al. [CoGeNT collaboration], "Results from a Search for Light-Mass Dark Matter with a P-type Point Contact Germanium Detector," arXiv:1002.4703 [astro-ph.CO].

[14] K. Nakamura et al. (Particle Data Group), J. Phys. G37, 075021 (2010).

[15] A. W. Strong, I. V. Moskalenko, T. A. Porter, G. Johannesson, E. Orlando and S. W. Digel, "The GALPROP Cosmic-Ray Propagation Code," arXiv:0907.0559 [astro-ph.HE].

[16] S. Albino, B. A. Kniehl and G. Kramer, "Fragmentation functions for light charged hadrons with complete quark flavour separation," Nucl. Phys. B 725, 181 (2005). arXiv:hep-ph/0502188. 